\title{
Articulo \\ Comportamiento epidemiológico de la COVID-19 en Costa Rica: del 6 de marzo al 31 de agosto del 2020
}

\author{
Epidemiological behavior of COVID-19 \\ in Costa Rica: from March 6 to August 31, 2020
}

Autores

Dr. Andrés Cairol Barquero, médico especialista en Administración de Servicios de Salud,

Coodinador del centro de entrenamiento en reanimación UNIBE

Dra. Wendy Morún Yargas,

Méalico cirujano

Dr. Luis Felipe Loaiza Sáenz,

Medico cirujano

Dr. Edward Segura Pérez,

Médico cirujano

Instructores del centro de entrenamiento en reanimación UNIBE.

Correspondencia: acairol@unibe.ac.cr

Palabras clave

Epidemiología, COVID-19, Costa Rica.

\section{Resumen \\ Introducción}

La nueva pandemia causada por el coronavirus, causante del COVID 19 tuvo su aparición en diciembre del 2019 en la localidad de Wuhan, China. El primer caso documentado fuera de las fronteras de Wuhan se presentó el 20 de enero en Corea del Sur, hasta llegar al 12 de marzo donde tuvo tal expansión que se pasó al estado de pandemia mediante un comunicado de la OMS.
El primer caso en Costa Rica se presentó el 6 de marzo y como fue el comportamiento usual aumentaron los casos y de asi mismo iniciaron estrategias por parte del gobierno costarricense para mitigar el impacto de la enfermedad. Materiales y métodos: recolección de los datos mediante la base de datos oficial del Ministerio de salud de Costa Rica. Para el análisis se utilizó Epidata ${ }^{\circledR}$, Excel y Epiinfo ${ }^{\circledR}$. Discusión: La tasa de letalidad o mortalidad en Costa Rica, es 2.84 veces más baja que la que presenta el continente. En Costa Rica, el odds de mortalidad asociado a la edad, aumenta por 
encima de 60 años, con un OR 5.48 (IC $95 \% 4.66$ - 6.44). El sexo femenino, presenta un OR de 1.47 (IC 95\% 0.97 - 2.24), mostrando datos estadísticamente no consistentes, debido al intervalo de confianza, por lo que no existe evidencia estadística para relacionar el sexo con la mortalidad. Conclusiones: El país ha logrado tasas de mortalidad bajas en relación a los otros países de la región de las américas. La enfermedad ha mostrado un crecimiento no lineal, de carácter multifactorial, como el comportamiento poblacional y el acatamiento a las disposiciones del ministerio de salud. La cantidad y aumento de casos en el área metropolitana hace pensar en transmisión comunitaria.

\section{Summary Introduction}

The new pandemic caused by the coronavirus, which causes COVID 19, begun in December 2019 in the town of Wuhan, China. The first documented case outside the borders of Wuhan occurred on January 20 in South Korea. In March 12, it had such an expansion that it became a pandemic through a statement sent by the WHO. The first case in Costa Rica occurred on March 6 and, as was the usual behavior, the cases increased and in the same way, strategies were initiated by the Costa Rican government to mitigate the impact of the disease. Materials and methods: data collection using the official database of the Ministry of Health of Costa Rica. Epidata ${ }^{\circledR}$, Excel and Epiinfo ${ }^{\circledR}$ were used for the analysis. Discussion: The fatality or mortality rate in Costa Rica is 2.84 times lower than the rest of the continent. In Costa Rica, the odds of mortality associated with age increase above 60 years, with an OR 5.48 (95\% CI 4.66 . 6.44). The female sex presents an OR of 1.47 (95\% CI 0.97 - 2.24), showing statistically inconsistent data, due to the confidence interval, so there is no statistical evidence to relate sex with mortality. Conclusions: The country has achieved low mortality rates in relation to other countries in the Americas region. The disease has shown a non-linear growth, due to multifactorial situations, such as population behavior and the obey of the government Instructions. The number and increase of cases in the metropolitan area suggests community transmission

\section{Introducción}

La nueva enfermedad por coronavirus del subtipo COVID 19 tuvo su aparición en diciembre del 2019 en China, específicamente en la localidad de Wuhan. Los primeros casos llamaron la atención debido a la aparición de pacientes con neumonías graves sin etiología aparente. El 31 de diciembre del 2019, la Organización Mundial de la Salud (OMS) informó sobre el estado de epidemia en Wuhan y, al día siguiente, se solicitó el cierre del Mercado de Mariscos de Huanuan, donde, según autoridades de China, surgió la pandemia. El 6 de enero, el Centro de Control de Enfermedades (CDC) declaró estado de emergencia de nivel 2 en China y recomendó al gobierno chino implementar medidas de control sobre entradas y salidas de China (1).

Fue para el 12 de enero del 2020, que autoridades de salud chinas determinaron la secuencia genética del nuevo coronavirus con miras a iniciar la formación de los kits diagnósticos. La propagación del virus sucedió de manera rápida, el primer caso fuera de Wuhan se documentó el 19 de enero y para el 20 de enero se reportó el primer caso fuera de China en Corea del Sur; posteriormente, el 21 de enero se presentó el primer caso en los Estados Unidos. Así continuó la transmisión del virus hasta que el 12 declara estado de pandemia a nivel mundial por el coronavirus. (1)

Según la página oficial del Ministerio de Salud de Costa Rica, el primer caso diagnosticado en nuestro país fue el 6 de marzo del 2020 en una mujer estadounidense de 49 años de edad que venía como turista. En el mismo vuelo del caso índice y su esposo, viajaban otras 152 personas, quienes fueron tamizadas como contactos del 
caso positivo. Luego, de manera paulatina, aumentaron los casos y, por ende, el país tuvo su respuesta epidemiológica.

El virus del síndrome respiratorio agudo severo tipo-2 (SARS-CoV-2), causante de COVID-19, taxonómicamente pertenece a la familia Coronaviridae. Esta familia se subdivide en cuatro géneros: Alphacoronavirus, Betacoronavirus, Gammacoronavirus y Deltacoronavirus $(2,3)$. Muchos coronavirus de esos cuatro géneros son los causantes de enfermedades en animales domésticos y, por lo tanto, son principalmente de interés veterinario (4). Los coronavirus de importancia médica conocidos hasta hoy son siete, y pertenecen a uno de los dos primeros géneros descritos (2). Desde el punto de vista ecoepidemiológico, esos virus se pueden clasificar en dos grupos: coronavirus adquiridos en la comunidad (o coronavirus humanos, $\mathrm{HCoV}$ ) y coronavirus zoonóticos
Haciendo un recuento de los eventos desde el primer caso positivo de COVID-19 en Costa Rica, el 6 de marzo del 2020, las decisiones sanitarias gubernamentales han estado dirigidas por el Ministerio de Salud, que ha alternado entre cierres totales y parciales de los establecimientos comerciales, restricción vehicular en diferentes horarios y suspensión de las clases presenciales de educación primaria y secundaria. La comisión nacional de emergencias declaró emergencia nacional, según el riesgo de cada cantón, clasificados como amarillo o anaranjado de acuerdo con el comportamiento epidemiológico del país. Esas medidas restrictivas se resumen en el siguiente diagrama.

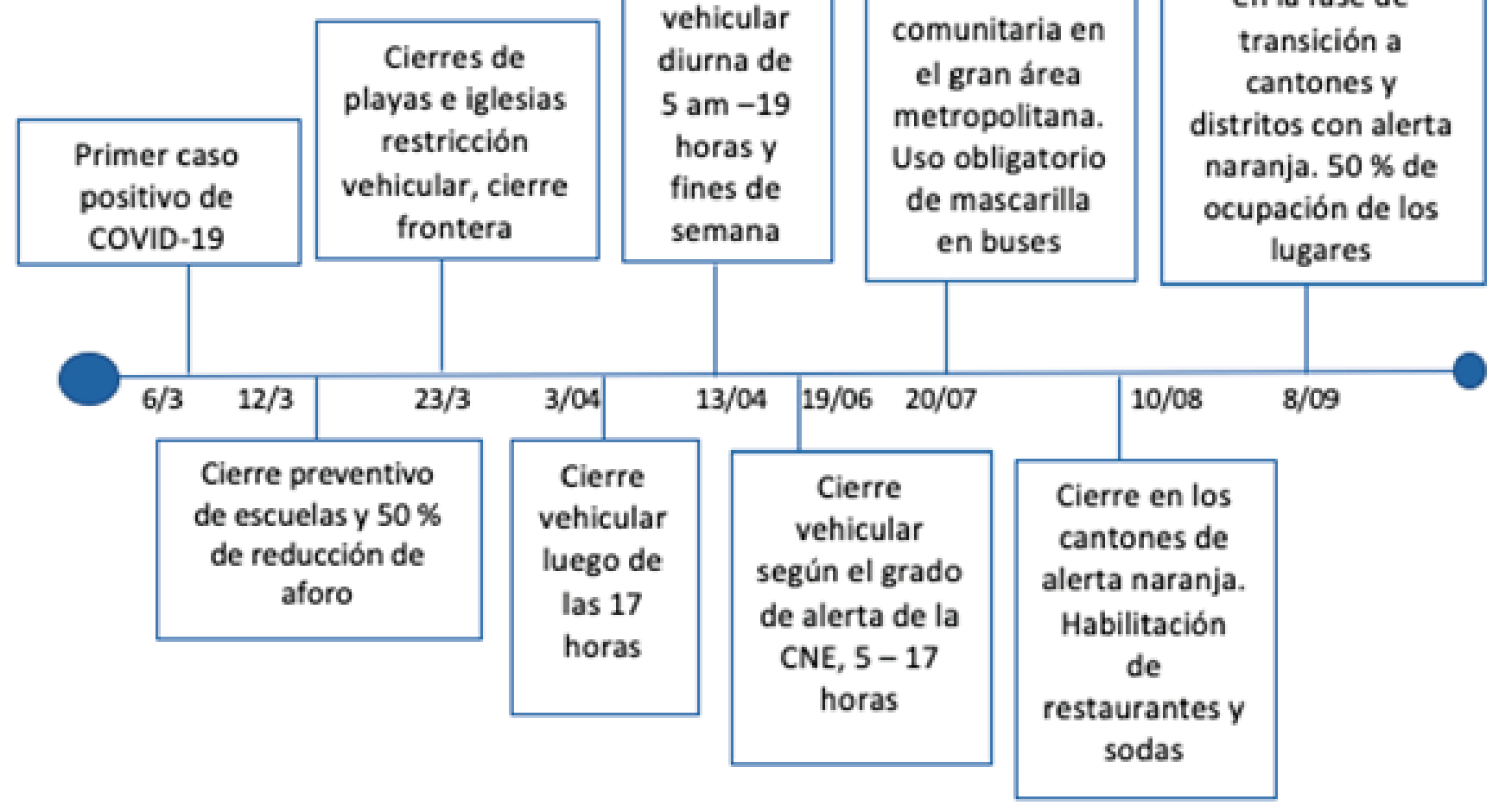




\section{Materiales y métodos}

Para la recolección de los datos, se consideró la base de datos oficial del Ministerio de Salud de Costa Rica, mediante la plataforma del Observatorio Geográfico en Salud, de la Universidad Estatal a Distancia (UNED). Para el análisis se utilizaron las herramientas informáticas epidata ${ }^{\circledR}$, Excel y la calculadora epidemiológica de Epiinfo ${ }^{\circledR}$.

Desde el 6 de marzo hasta el 31 de agosto del 2020, en Costa Rica, se diagnosticaron 41287 personas con la COVID-19, de esa cantidad el $48.71 \%$ eran mujeres y $51.28 \%$, hombres. El promedio de edad de las mujeres es de 49.04 años y de los hombres de 50.2 años.

Tabla 1. Distribución epidemiológica de casos de COVID - 19 en Costa Rica, del 3 de marzo al 31 de agosto del 2020

Variable Valor

\section{Género}

Mujeres (\%) 20114 (48.71\%)

Hombres (\%) 21173 (51.28\%)

Edad (años)

Edad promedio
(DE)

Rango de edad

0 - 10 años

11 - 20 años

$21 \_30$ años

31 - 40 años

41 - 50 años

51 - 60 años

61 - 70 años

71 - 80 años

81 - 90 años

$>91$ años

\section{$50.24(29.65)$}

0 - 124

2153

3566

9642

10051

6974

5157

2280

1059

496

117
Fuente: base de datos del Ministerio de Salud de Costa Rica

La distribución por edad según el sexo muestra que la mayor dispersión se presenta entre los hombres; la media de edad es muy similar entre ambos sexos, sin embargo, el caso de mayor edad correspondió al sexo masculino. Adicionalmente, el percentil 25 en las mujeres fue de 24,5 años, en los hombres de 25 años; el percentil 75 en las mujeres fue de 73,5 años y en los hombres de 75 años, lo cual muestra que los datos en ambos grupos son muy similares. (Gráfico 1)

Gráfico 1. Distribución por la edad según el sexo en los casos positivos de COVID- 19 en Costa Rica, del 6 de marzo al 31 de agosto del 2020

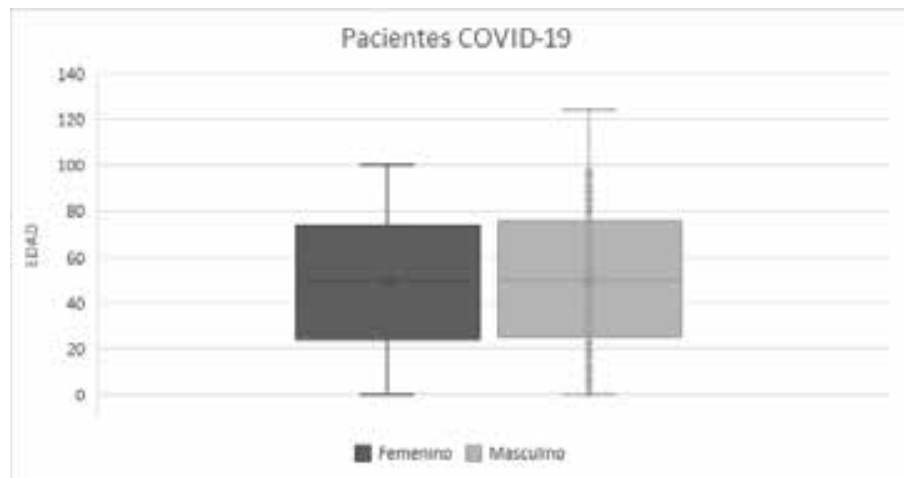

Fuente: base de datos del Ministerio de Salud de Costa Rica

El promedio de casos de los 179 días, entre el 6 de marzo al 31 de agosto del 2020, es de 243 por día (IC 95 \% 200- 287). Durante el 50 \% de esos días, se diagnosticaron entre 35 y 448 casos de COVID -19 (recorrido intercuartílico). (Gráfico 2)

Gráfico 2. Distribución del comportamiento de los casos diarios y acumulados de COVID-19 en Costa Rica, del 6 de marzo al 31 de agosto del 2020

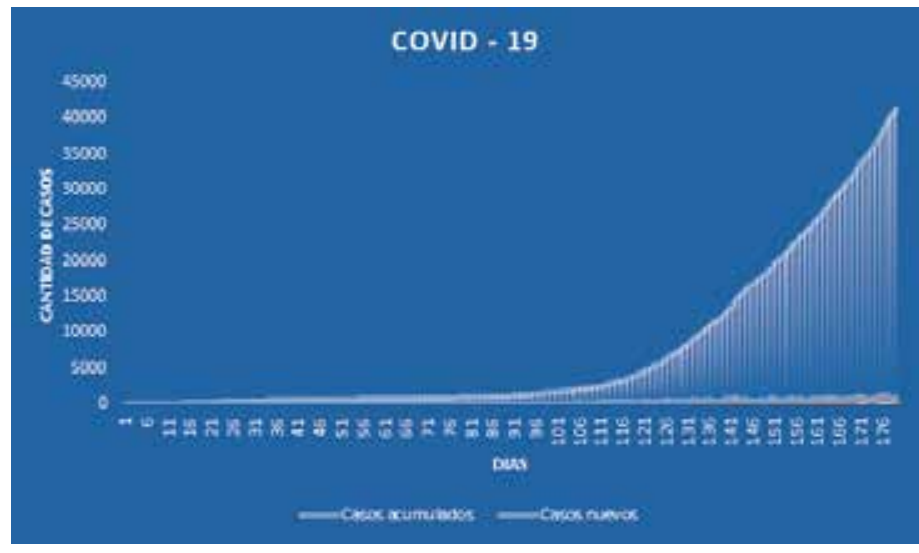

Fuente: base de datos del Ministerio de Salud de Costa Rica 
Una de las características de esta pandemia es el comportamiento no lineal de la cantidad de casos positivos por día, que se evidencia en el coeficiente de variación de $310 \%$. Eso demuestra, como muchas otras pandemias, que el comportamiento es exponencial (Gráfico 3). Por esta razón, existen varias metodologías para el pronóstico de los datos, como el llamado SIR (susceptibles, infectados y recuperados) (5) que pretende proveer una herramienta para estimar el comportamiento de la cantidad de casos, cuántos se han recuperado y cuántos son susceptibles al virus con la finalidad de que el sector salud pueda prepararse para la atención de la pandemia. Grafico 3. Distribución diaria de casos positivos de COVID-19 en Costa Rica, del 6 de marzo al 31 de agosto del 2020

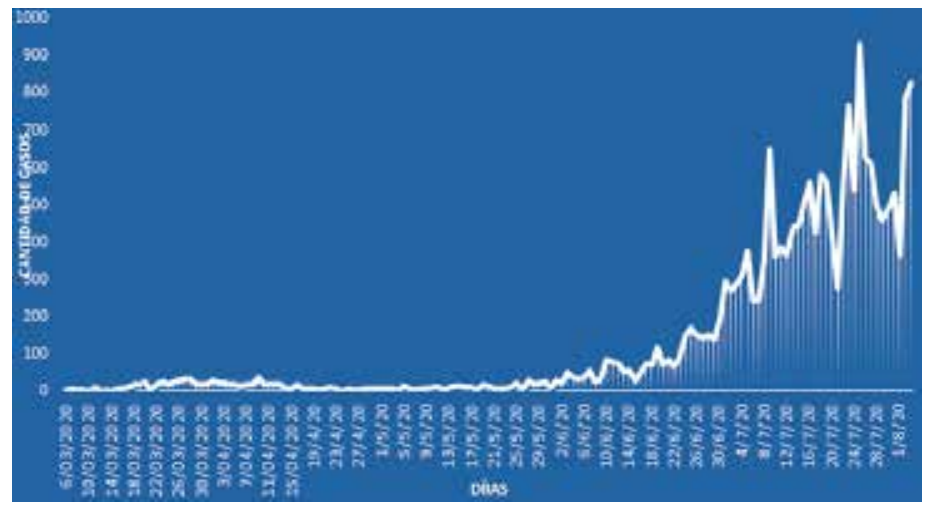

Fuente: Base de datos del Ministerio de Salud de Costa Rica

Para complementar el análisis se calculó el número de reproducción efectivo (Rt), que estima cuántos casos susceptibles pueden ser infectados en promedio por una persona que porta el virus (5), así, cuanto mayor sea este valor, mayor probabilidad existirá de que al no aislar al paciente positivo, la cantidad de personas que son susceptibles pueda aumentar. Este indicador considera el promedio de casos de los últimos 14 días para compararlo con el valor del día 15 en específico. Por esta razón, y en virtud de la naturaleza del indicador, el $\mathrm{R} \_t$ inicial corresponde al 19 de marzo del 2020, y el valor fue de 3.39 .
Esos datos permiten interpretar que en promedio un paciente positivo tiene capacidad para infectar a 3.39 personas susceptibles. El valor máximo de $\mathrm{R} \_t$, 4.18 , se obtuvo el 20 de marzo del 2020 y el valor mínimo de 0,09 se tuvo el 24 de abril del 2020. Idealmente, para lograr un control sostenido de la tasa de reproducción del virus y disminuir la cantidad de pacientes nuevos, el valor del $R \_t$ debe estar por debajo de 1 , de manera sostenida, es decir, debe tener en promedio menos de un paciente positivo por cada positivo nuevo diagnosticado. (Gráfico 4)

Gráfico 4. Comportamiento del valor del $\mathrm{R} \_\mathrm{t}$ de la pandemia de COVID-19 en Costa Rica, desde el 6 de marzo al 31 de agosto del 2020

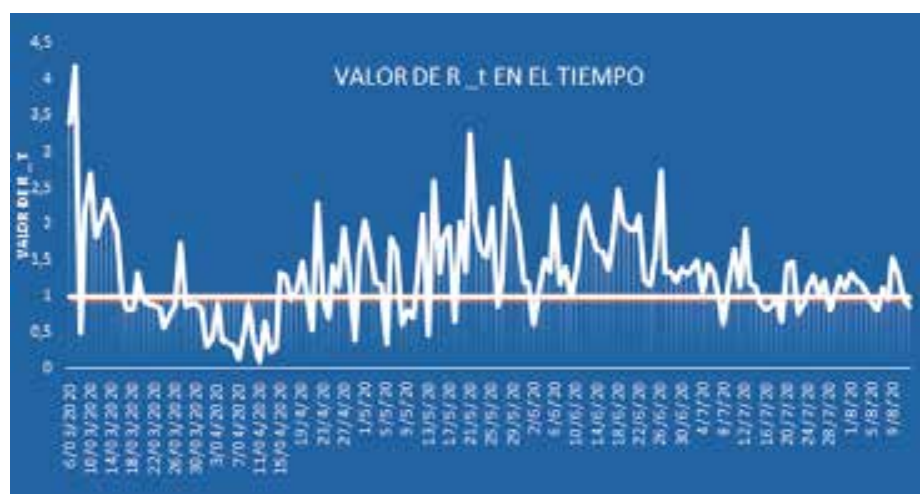

Fuente: calculado con los datos de la base de datos del Ministerio de Salud de Costa Rica

Como toda enfermedad que tiene potencial pandémico, una de las preocupaciones que ha surgido con la COVID-19 es el efecto que tendrá sobre el sector salud, en específico a nivel hospitalario. Una de las maneras de evidenciar ese impacto es con la tasa de hospitalizados por cada 100 pacientes positivos con COVID-19. Al inicio de la pandemia tuvimos tasas de 4 a 6 pacientes hospitalizados por cada 100 pacientes positivos con COVID-19, no obstante, conforme fue avanzando la enfermedad y la cantidad de pacientes diagnosticados, la tasa promedio de hospitalizados fue de 1.8 por cada 100 positivos (Gráfico 5). 
Gráfico 5. Tasa de hospitalizados por cada 100 pacientes positivos con COVID-19 en Costa Rica, desde el 3 de marzo al 31 de agosto del 2020

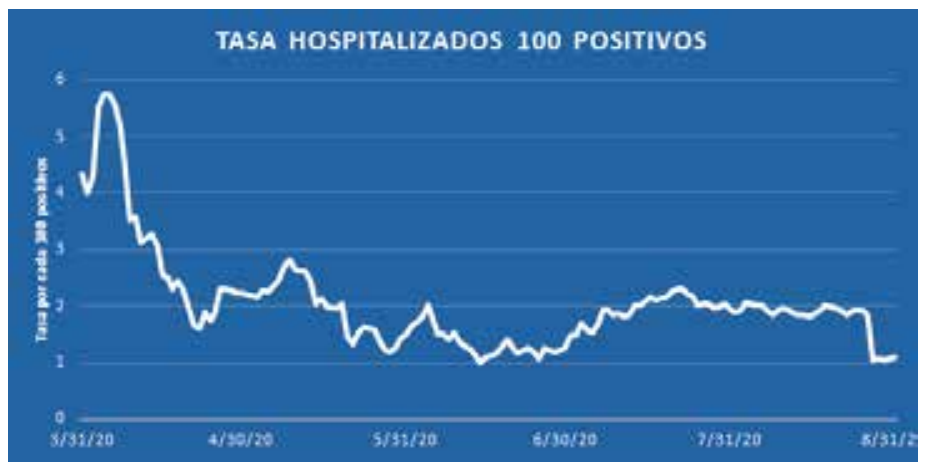

Fuente: calculado con los datos del Ministerio de Salud de Costa Rica.

Durante el periodo estudiado, se hospitalizaron 450 personas con COVID-19 lo que representa un $1.09 \%$ de los pacientes diagnosticados y la tasa de pacientes ingresados en la Unidad de Cuidados Intensivos (UCI) por su gravedad clínica fue de 0.37 por cada 100 pacientes positivos, de modo que osciló en $32 \%$ de los pacientes que ingresaron a un hospital. La tasa de mortalidad fue de 10,18 por cada 1000 pacientes positivos, lo que corresponde a un 1.02 $\%$ del total de pacientes.

La mejor manera de visualizar el comportamiento de infectados y fallecidos por provincia, es mediante el cálculo de las tasas de positivos por cada 1000 personas y la tasa de mortalidad o letalidad por cada 100 positivos.

La provincia que muestra la tasa de positivos más alta por cada 1000 personas es San José, 15.11 x 1000 personas, y la provincia con menor tasa de positivos es Guanacaste con $2.44 \times 1000$ personas.

La provincia con la mayor tasa de letalidad o mortalidad por cada 100 personas positivas es San José con 1.21 x 100 positivos y Limón es la provincia con la menor tasa de letalidad, $0.56 \mathrm{x}$ 100 positivos.
Distribución de la tasa de mortalidad por cada 100 positivos, y la tasa de positivos por cada 1000 personas de las provincias de Costa Rica por COVID-19

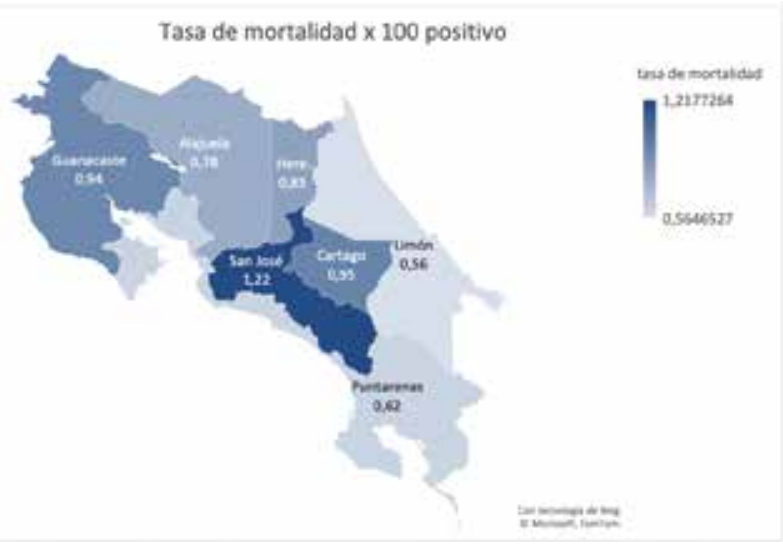

Distribución de los casos positivos por cada 1000 personas, y la tasa de positivos por cada 1000 personas de las provincias de Costa Rica por COVID-19

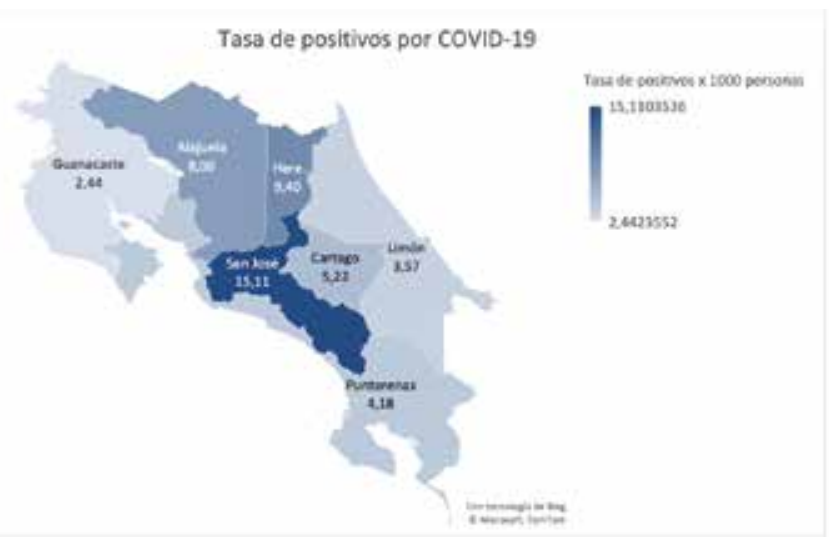

Fuente: elaboración propia con los datos del Ministerio de Salud, Costa Rica

La edad internacionalmente ha demostrado ser un factor de riesgo de mortalidad por COVID-19 (6). En nuestro país, luego de analizar la base de datos completa de COVID-19, con 41287 datos, la edad demostró ser un factor de riesgo, por encima de los 60 años, con un OR 5.48 (IC $95 \%$ 4.66 - 6.44), el grupo de edad con mayor riesgo son las personas mayores de 80 años, con un OR 17.29 (IC 95\% 15.33- 19.51). Por debajo de los 60 años, el OR es menor a 1. 
Tabla 2. Odds ratio mortalidad según el intervalo de edad de los pacientes de COVID-19, del 3 de marzo al 31 de agosto del 2020

Odds ratio mortalidad por COVI-19 por rango de edad

\begin{tabular}{ccccc}
\hline \multicolumn{6}{c}{ Odds ratio mortalidad por COVl-19 por rango de edad } \\
\hline Rango edad & Casos & Fallecldos & OR & IC 95\% \\
\hline 0- 20 años & 5240 & 5 & 0.09 & $0.03-0.22$ \\
$21-40$ años & 22385 & 20 & 0.08 & $0.05-0.13$ \\
41- 60 años & 14381 & 96 & 0.65 & $0.52-0.81$ \\
61- 80 años & 4064 & 227 & 5.48 & $4.66-6.44$ \\
Mayores de 80 años & 717 & 134 & 17.29 & $15.33-19.51$ \\
\hline
\end{tabular}

OR: Odds ratio, IC: intervalo de confianza.

Fuente: Calculado con datos de la base de COVID-19 del Ministerio de Salud de Costa Rica.

\section{Discusión}

Según el informe 23 de la Organización Panamericana de la Salud (OPS), con corte al 31 de agosto del 2020 (7), se ha diagnosticado en la región un total de 13356411 personas confirmadas con COVID-1 y 467149 muertes por la enfermedad, lo que corresponde a una tasa de letalidad de $349 \times 10000$ personas o lo que es lo mismo de 3.49 por 1000 pesonas con COVID- 19. La tasa de letalidad o mortalidad en Costa Rica, es 2.84 veces más baja que la que presenta el continente en total. Los países de Centroamérica que mostraron mayor tasa de mortalidad por COVID-19 fueron Panamá 41 x 100000 habitantes, Honduras 16 por cada 100 000 habitantes y Guatemala 14 x 100000 habitantes, mientras que en Costa Rica es de $9.35 \times 100000$ habitantes. La tasa de pruebas positivas en Costa Rica es de 288.24 x 1000 de las pruebas realizadas.

La relación entre la edad y el riesgo de mortalidad por COVID-19 en Costa Rica es claro, mediante el análisis del inicio de la enfermedad en Whuhan, China (8), encontraron que por encima de los 40 años se iniciaba la probabilidad de morir por COVID-19, con un OR 5.6 (IC $95 \% 0.69$ - 6.5), sin embargo, el dato es estadísticamente no adecuado por el intervalo de confianza, por lo que los pacientes mayores de 50 años tuvieron un OR 6.76 (IC 95\% 2.89- 15.16), mientras que en Costa Rica, el Odds de mortalidad asociado a la edad, aumentó por encima de 60 años con un OR 5.48 (IC $95 \% 4.66-6.44$ ).

La correlación de la probabilidad de mortalidad según el sexo, en Wuhan China (8) presentó que el OR para sexo femenino fue de 1.85 )IC $95 \%$ 1.59- 2.12) lo que muestra un leve aumento en cuanto a la probabilidad de fallecer por COVID-19 en comparación con los hombres; mientras que en Costa Rica el sexo femenino, presentó un OR de 1.47 (IC 95\% $0.97-2.24$ ), mostrando datos estadísticamente no consistentes debido al intervalo de confianza, por lo que no existe evidencia estadística para relacionar el sexo con la mortalidad.

\section{Conclusiones}

Desde la última pandemia por un virus respiratorio en el 2009, que mató a 284000 personas en todo el mundo, no se tenía la experiencia para manejar una enfermedad con una cualidad de transmitirse tan rápidamente, lo que ha provocado un importante impacto en todos los países y por supuesto también en Costa Rica. Desde el primer caso el 6 de marzo del 2020, el Ministerio de Salud, ente rector y el prestatario de la atención médica, la Caja Costarricense del Seguro Social, volcaron sus recursos a la contención de la pandemia del COVID-19. Así, al 31 de agosto del 2020 se habían contagiado 41287 personas y fallecido lastimosamente 436, y 450 personas habían pasado por la unidad de cuidados intensivos en los hospitales del sector salud público costarricense.

El país ha logrado tasas de mortalidad bajas en relación con los otros países de la región 
latinoamericana. La enfermedad ha mostrado en Costa Rica un crecimiento no lineal, lo cual ha dependido de muchos factores, uno de ellos, el comportamiento de la población y el acatamiento a las disposiciones del Ministerio de Salud. En las últimas semanas antes del 31 de agosto, la cantidad de casos positivos fue en aumento, sobre todo, en el área metropolitana que muestra transmisión comunitaria del virus.

Las estimaciones mediante modelos estadísticos, como el SIR, han tenido dificultades para estimar con exactitud el comportamiento del virus en el país. No obstante, conocer el comportamiento epidemiológico de la COVID-19, como lo expone este trabajo de investigación, le permite al sector salud tener un panorama más claro y así poder responder adecuadamente a una enfermedad nueva de la cual se aprende todos los días. Actualmente no se cuenta con ninguna publicación científica que describa el comportamiento de la enfermerdad pandémica en Costa Rica, por lo que esta investigación, pretende sentar las bases para futuras investigaciones que de manera restrospectiva al acabar la pandemia, estudie la totalidad de los datos.

\section{Bibliografía}

1. Bandyopadhyay A., Chatterjee D., Ghosh K. y Sarkar P. COVID 19: Evaluación epidemiológica y genética del hospedador. Revista Asiática de Ciencias Médicas. 11 (3), 71-76, 2020.

2. Pal M, Berhanu G, Desalegn C, Kv R. Severe acute respiratory syndrome coronavirus-2 (SARS-CoV-2): An update. Cureus, 2020.

3. Chan JF-W, Kok K-H, Zhu Z, Chu H, To KKW, Yuan S, et al. Genomic characterization of the 2019 novel human-pathogenic coronavirus isolated from a patient with atypical pneumonia after visiting Wuhan. Emerg Microbes Infect. 9:221-236, 2020.
4. American Veterinary Medical Association (AVMA). Coronavirus: Detailed taxonomy. Schaumburg, IL: AVMA. Acceso 4 de abril de 2020 .

5. Navarro-Robles E, Martínez-Matsushita L, López-Molina R, Fritz-Hernández J, Flores-Aldana B, Mendoza-Pérez J. Modelo para estimación del comportamiento epidémico de la influenza A (H1N1) en México. Revista Panamericana de Salud Pública. 31(4):269-274, 2012

6. The factors predicting pneumonia in COVID-19 patients: preliminary results of a university hospital in Turkey. Turkish Journal of Medical Sciences. 2020

7. COVID-19 - Respuesta de la OPS/OMS Reporte 23 (31 de agosto de 2020) - OPS/OMS | Organización Panamericana de la Salud [Internet]. Paho.org. [cited 11 September 2020].

8. The factors predicting pneumonia in COVID-19 patients: preliminary results of a university hospital in Turkey. TURKISH JOURNAL OF MEDICAL SCIENCES. 2020. 